\title{
Tuberculosis in Biologic-naïve Patients With Rheumatoid Arthritis: Risk Factors and Tuberculosis Characteristics
}

\author{
Johanna Karlsson Sundbaum ${ }^{1}$, Elizabeth V. Arkema ${ }^{2}$ (D) Judith Bruchfeld ${ }^{3}$, Jerker Jonsson ${ }^{4}$ (D), \\ Johan Askling 5 , and Eva Baecklund ${ }^{6}$
}

\begin{abstract}
Objective. To investigate risk factors and characteristics of active tuberculosis (TB) in biologic-naïve patients with rheumatoid arthritis (RA).

Methods. We conducted a population-based case-control study using the Swedish Rheumatology Quality Register, the National Patient Register, and the Tuberculosis Register to identify RA patients with active TB and matched RA controls without TB between 2001-2014. Clinical data were obtained from medical records. TB risk was estimated as adjusted OR (aOR) with 95\% CI using univariate and multivariable logistic regression analyses.

Results. After validation of diagnoses, the study included 31 RA patients with TB and 122 matched RA controls. All except 3 cases had reactivation of latent TB. Pulmonary TB was most prevalent (84\%). Ever use of methotrexate was not associated with increased TB risk (aOR 0.8, 95\% CI 0.3-2.0), whereas ever treatment with leflunomide (aOR 6.0, 95\% CI 1.5-24.7), azathioprine (aOR 3.8, 95\% CI 1.1-13.8), and prednisolone (PSL; aOR 2.4, 95\% CI 1.0-6.0) was. There were no significant differences between maximum dose of PSL, treatment duration with PSL before TB, or cumulative dose of PSL the year before TB diagnosis between cases and controls. Obstructive pulmonary disease was associated with an increased TB risk (aOR 3.9, 95\% CI 1.5-10.7).

Conclusion. Several RA-associated factors may contribute to increased TB risk in biologic-naïve patients with RA, making the risk of TB activation difficult to predict in the individual patient. To further decrease $\mathrm{TB}$ in patients with RA, the results suggest that screening for latent TB should also be considered in biologic-naïve patients.
\end{abstract}

Key Indexing Terms: biologic-naïve, rheumatoid arthritis, risk factors, tuberculosis

Shortly after the introduction of tumor necrosis factor inhibitors (TNFi) in the treatment of rheumatoid arthritis (RA), an increase in the risk of tuberculosis (TB), in particular of reactivation of

No specific funding was received from any bodies in the public, commercial, or not-for-profit sectors to carry out the work described in this article.

${ }^{1}$ J. Karlsson Sundbaum, PhD, Assistant Professor, Rheumatology Unit, Department of Medical Sciences, Uppsala University, Uppsala, and Department of Health Sciences, Luleå University of Technology, Luleå; ${ }^{2}$ E.V. Arkema, PhD, Assistant Professor, Clinical Epidemiology Division, Department of Medicine Solna, Karolinska Institutet, Stockholm; ${ }^{3} J$. Bruchfeld, PhD, Senior Consultant, Department of Infectious Diseases, Karolinska University Hospital, Stockholm, and Division of Infectious Diseases, Department of Medicine Solna, Karolinska Institutet, Stockholm; ${ }^{4}$ J. Jonsson, PhD, Senior Consultant, Public Health Agency of Sweden, Stockholm; J. Askling, PhD, Professor, Clinical Epidemiology Division, Department of Medicine Solna, Karolinska Institutet, Stockholm, and Rheumatology Division, Department of Medicine, Karolinska Institutet, Stockholm; ${ }^{6}$ E. Baecklund, $\mathrm{PhD}$, Associate Professor, Rheumatology Unit, Department of Medical Sciences, Uppsala University, Uppsala, Sweden.

The authors declare no conflicts of interest.

Address correspondence to Dr. J. Sundbaum, Rheumatology Unit, Department of Medical Sciences, Uppsala University, SE-751 85 Uppsala, Sweden.Email:johanna.sundbaum@ltu.se.

Accepted for publication March 11, 2021. latent TB infection (LTBI), was observed. ${ }^{1}$ Ensuing guidelines recommending screening and treatment for LTBI before starting treatment with TNFi and other biologic (b) disease-modifying antirheumatic drugs (DMARDs) have been followed by a decrease of active TB among bDMARD-treated patients with RA. ${ }^{2,3}$ However, the TB risk in biologic-naïve patients with RA appears to remain several-fold higher compared to the general population. In a previous study, we showed that the rate of $\mathrm{TB}$ in bDMARD-treated patients with RA decreased following the initiation of pretreatment TB screening in Sweden, but no similar decline in risk was observed among the biologic-naïve patients with RA. ${ }^{4}$ The risk in this group remained 4 times higher than the general population in Sweden. Notably, most TB cases in contemporary patients with RA occur in biologic-naïve patients. ${ }^{5,6}$ It is therefore reasonable that efforts to further decrease the TB risk in RA should also incorporate the group of biologic-naïve patients. To do this, a better understanding of TB risk factors and characteristics in this patient group is essential, yet consistent results are largely lacking (studies summarized in Supplementary Table 1, available with the online version of this article)..$^{7-20}$

We aimed to assess risk factors for active TB infection in biologic-naïve patients with RA and to describe the clinical characteristics and outcomes of the clinical TB manifestations. We therefore performed a matched population-based case-control 
study using national registers in combination with data collection from medical records.

\section{METHODS}

Patients with $R A$. We identified a national population-based RA cohort from the National Patient Register (NPR) using registered inpatient care from 1969 to 2014 and outpatient, nonprimary care from 2001 to 2014, and from the Swedish Rheumatology Quality Register (SRQ) between 1997-2014. The registration of inpatient care in the NPR is over $99 \%$ complete since 1987 and the outpatient specialist nonsurgical care component is over $80 \%$ complete. ${ }^{21,22}$ Patients were included if they were $\geq 18$ years old at RA diagnosis and had at least 2 visits with an International Classification of Diseases (ICD) code for RA (ICD-10: M05, M06.0, M06.2, M06.8, M06.9, M12.3; ICD-9: 714A, 714B, 714C, 714W, 719D; ICD-8: $712.10,712.20,712.38)$ in the NPR and/or SRQ. The patients were required to have at least 1 specialist visit to a rheumatology or internal medicine department in the NPR and at least 1 visit in outpatient care. With this definition of RA, it has been shown that $>90 \%$ of the identified patients fulfilled current criteria for RA. ${ }^{23}$ The RA diagnoses in the medical records were validated against the American College of Rheumatology (ACR) 1987 or the ACR/European League Against Rheumatism (EULAR) 2010 RA criteria. To identify the patients with RA unexposed to bDMARDs, we used treatment data from SRQ. Data on exposure to bDMARDs are entered by clinicians in the SRQ, and the registry covers approximately $90 \%$ of the bDMARD-treated Swedish patients with RA.

Cases with TB. Cases were patients with RA unexposed to bDMARDs with a first TB diagnosis after the second discharge listing RA in the NPR, identified by linking the RA population and the Swedish Tuberculosis Register from 2001-2014. All forms of active TB are notifiable according to Swedish law, and clinicians are obliged to report to the Swedish Tuberculosis Register any individual they start on TB treatment, whether it be for verified or suspected TB. Laboratories report mycobacterial culture-positive patients in the same system, linked to the clinician's report by the patient's unique personal number. If the laboratory report is not followed by a clinical report within a few weeks, the treating physician is asked to complete the report. The coverage of TB cases confirmed by culture for the register is therefore close to $100 \%$.

Controls free from $T B$. For each bDMARD-unexposed RA case with TB, 4 RA controls without TB were identified from the RA population who were living in Sweden and who had not been exposed to bDMARDs before the TB diagnosis of the corresponding case. Controls had to be free from any registered TB diagnosis in the NPR and the TB register, and were matched on sex, year of birth ( \pm 5 yrs), year of first RA diagnosis in the NPR, and region of residence at the time of the $\mathrm{TB}$ diagnosis of their corresponding case.

Exposures. Clinical data of cases and controls were collected from medical records and included information about RA disease, smoking habits, and comorbidities known at any time before TB diagnosis for cases, and before TB diagnosis of the matched case for controls. Data on RA disease activity at visits 6 months ( \pm 1 month) before TB diagnosis and at TB diagnosis $( \pm 1$ month) were obtained, together with data on RA disease activity during TB treatment and outcome of TB treatment for the cases. RA disease activity was considered increased if (1) a note in the medical records mentioned increased RA disease activity, such as more affected joints compared to last visit; (2) the patient had received several local corticoid injections; or (3) there was a change in therapy due to increased disease activity.

Data on exposure to corticosteroids and any conventional synthetic (cs)DMARD, including methotrexate (MTX), sulfasalazine, hydroxychloroquine/chloroquine, leflunomide (LEF), cyclosporine, azathioprine (AZA), cyclophosphamide, penicillamine, myocrisine, and auranofin, for $\geq 4$ consecutive weeks from RA diagnosis until TB diagnosis and at specific timepoints (i.e., 12 months, 9 months, 6 months, and 3 months) before TB diagnosis were obtained, together with treatment duration and maximum dose. For MTX and prednisolone (PSL), information on cumulative dose the year before TB diagnosis and the exposure to doses $\geq 15 \mathrm{mg}$ of PSL $\geq 1$ month at any time during the course of RA, as well as during the 12 months and 6 months preceding TB diagnosis, was also collected.

Established epidemiological risk factors for TB were assessed. These included former TB infection or known exposure to TB in the family (parents or siblings with diagnosed TB), occupational history, and a history of living abroad in high-endemic areas. To be born before 1950 was regarded as a risk factor in descriptive analyses, but not in risk assessments as cases and controls were matched for age. If the patient had been screened for LTBI, the type of screening test and outcome were collected.

Statistical analysis. For comparative analyses between cases and controls, $t$ tests and Mann-Whitney $U$ tests were used for continuous data, and chi-square and Fisher exact tests were used for categorical data. $P$ values $<0.05$ were considered statistically significant. Age- and sex-adjusted logistic regression models were used to estimate the relative risk for TB expressed as adjusted OR (aOR) with 95\% CI. Analyses of PSL treatment were additionally adjusted for DMARD use. We used a complete case analysis approach, meaning that for each exposure patients with missing values were excluded from the respective analysis. Data analyses were conducted using SPSS version 25 for Windows (IBM Corp.).

This study complies with the Declaration of Helsinki and was approved by the regional ethics committee in Uppsala, Sweden (2015/306). According to the ethical approval, no written informed consent from the patients was needed.

\section{RESULTS}

We initially identified 42 biologic-naïve RA patients with $\mathrm{TB}$ and 164 matched biologic-naïve RA controls without TB from the register data during the 2001-2014 study period. After exclusions, mainly due to missing or incomplete medical records or because patients did not fulfill RA criteria (Supplementary Figure 1, available with the online version of this article), we finally included 31 cases with verified RA and active TB and 122 matched RA controls without TB. Fifteen (48\%) of the cases and 70 (57\%) of the controls were women. Table 1 summarizes the RA characteristics and comorbidities of cases and controls.

$T B$ characteristics and screening. The mean age at TB diagnosis was 73.2 years, with TB diagnosed on average $15.8(0-58)$ years after the RA diagnosis. Most patients reported typical TB symptoms such as weight loss, cough, fever, and night sweats, followed by a TB diagnosis on average 15 weeks after onset of symptoms. Pulmonary TB was most prevalent $(84 \%)$. There were no drug-resistant strains, and most patients had unique strains indicating reactivation of LTBI. A possible acquisition of new infection abroad (United States, Thailand, and the Baltic countries) was reported in the Swedish Tuberculosis Register in 3 cases, whereas all other cases were regarded as reactivation of LTBI. The patients tolerated TB treatment well without any reported serious adverse events. Overall, the prognosis of TB was good and $94 \%$ of the patients fulfilled the treatment course, whereas 1 patient died of miliary TB and 3 patients died from other medical conditions before completing TB therapy (Table 2).

Early screening for LTBI with tuberculin skin test (TST) was performed in only 2 of the patients- 1 case (who later developed active TB) and 1 control-both with identified epidemiological risk factors for TB exposure. Both had negative TST results and were not given preventive $\mathrm{TB}$ treatment. 
Table 1. Characteristics of TB cases with biologic-naïve RA and matched RA controls.

\begin{tabular}{lcc}
\hline & TB Cases, $\mathrm{n}=31$ & Controls, $\mathrm{n}=122$ \\
\hline Sex, female, $\mathrm{n}(\%)$ & $15(48)$ & $70(57)$ \\
Age at RA diagnosis, yrs, mean \pm SD (min-max) & $57.4 \pm 18.1(17-82)$ & $55.8 \pm 15.8(13-81)^{\mathrm{a}}$ \\
Age at TB diagnosis, yrs, mean \pm SD (min-max) & $73.2 \pm 10.2(45-87)$ & - \\
RA diagnosis and treatment in Sweden & $31(100)$ & $121(99)^{\mathrm{b}}$ \\
RF positivity & $26(84)$ & $84(69)$ \\
Missing & $2(7)$ & $7(6)$ \\
Anti-CCP positivity & $7(22)$ & $24(19)$ \\
Missing & $20(65)$ & $77(63)$ \\
Treatment during the course of RA ${ }^{\mathrm{c}}$ & & $117(96)$ \\
DMARD & $30(97)$ & $97(80)$ \\
DMARD during the year before TB & $26(84)$ & $63(54)$ \\
CS & $23(74)$ & $52(44)$ \\
CS during the year before TB & $11(50)$ & $5(4)$ \\
CS missing & $0(0)$ & $11(9)$ \\
Comorbidities known at diagnosis of TB & \\
Cancer & $4(14)$ & $15(12)$ \\
Diabetes & $3(10)$ & $11(9)$ \\
COPD or asthma & $9(29)$ & $5(4)$ \\
Comorbidities missing & $0(0)$ & $47(38)$ \\
Ever smoker & $16(52)$ & $42(34)$ \\
Missing & $6(20)$ & \\
\hline
\end{tabular}

Values are expressed as n (\%) unless stated otherwise. ${ }^{a}$ Missing data from 5 patients. ${ }^{b}$ One patient diagnosed in the United States. ${ }^{\mathrm{c}}$ Treatment for $\geq 4$ consecutive weeks from RA diagnosis until TB diagnosis. ${ }^{\mathrm{d}}$ For controls at diagnosis of the corresponding case. CCP: cyclic citrullinated peptide; COPD: chronic obstructive pulmonary disease; CS: corticosteroid; DMARD: disease-modifying antirheumatic drug; RA: rheumatoid arthritis; RF: rheumatoid factor; TB: tuberculosis.

Table 2. Characteristics of TB patients with biologic-naïve RA.

\begin{tabular}{lc}
\hline & TB Cases, $\mathrm{n}=31$ \\
\hline Characteristics of TB & \\
$\quad$ Duration of symptoms before TB diagnosis, \\
weeks, mean \pm SD $($ min-max $)$ & $15 \pm 9(2-41)^{\mathrm{a}}$ \\
Pulmonary TB & $26(84)$ \\
Extrapulmonary $\mathrm{TB}^{\mathrm{b}}$ & $8(26)$ \\
Miliary TB & $2(6)$ \\
Diagnosis of TB & $7(23)$ \\
Smear-positive TB & \\
Bacteriologically confirmed TB by culture & $\mathrm{d}$ \\
Clinically diagnosed TB $^{\mathrm{b}}$ & $20(65)$ \\
Other & $6(19)$ \\
Treatment of TB & $5(16)$ \\
Drug resistance & \\
Treatment completed & $0(0)$ \\
Died of TB & $27(94)$ \\
\hline
\end{tabular}

Values are expressed as n (\%) unless stated otherwise. ${ }^{a}$ Missing data from 5 patients. ${ }^{b}$ Five patients with pulmonary and extrapulmonary localization. ${ }^{c}$ All smear-positive tests were confirmed by culture. ${ }^{\mathrm{d}}$ Includes 7

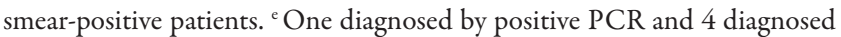
by positive pathological-anatomical diagnosis. ${ }^{\mathrm{f}}$ Standard treatment in most cases 6 months with isoniazid, rifampicin, pyrazinamide, and ethambutol (at initiation). RA: rheumatoid arthritis; TB: tuberculosis.

$R A$-associated risk factors for $T B$. MTX was the most common DMARD, used at any time in $68 \%$ of the TB cases and $74 \%$ of the controls. Treatment with MTX was not associated with an increased TB risk (aOR 0.8, 95\% CI 0.3-2.0), and there were no significant differences between cases and controls regarding maximum dose, treatment time, or cumulative dose of MTX in the last year before TB onset (Table 3 and Table 4). In contrast, although used in only a minority of the patients, treatment during the last year before TB onset with LEF was associated with increased TB risk (aOR 8.6, 95\% CI 1.5-50.5), as well as treatment with AZA (aOR 17, 95\% CI 1.6-161). Analyses comparing other DMARDs did not reveal any significant differences between cases and controls (Table 3).

Treatment with oral corticosteroids (PSL) during the course of RA was associated with an aOR for TB of 2.5 (95\% CI 1.0-6.0; Table 3). No significant differences were identified between PSL-treated cases and controls in terms of maximum dose ever of PSL, treatment duration before $\mathrm{TB}$, treatment during the last year before TB, or cumulative dose of PSL during the last year before diagnosis of TB. Likewise, there were no associations with TB and PSL treatment $\geq 15 \mathrm{mg}$ with a duration of $\geq 1$ month ever or during the last 12 months (Table 5), and 6 months, before diagnosis of TB (data not shown).

An increase in RA disease activity was reported in $20 \%$ of cases compared to $7.3 \%$ of controls $(P=0.07)$ during the 6 months preceding the TB diagnosis. A majority of these cases also continued to have active RA disease during TB treatment. Obstructive pulmonary disease was linked to an increased TB risk (aOR 3.9, 95\% CI 1.5-10.7; Table 3). No other evaluated comorbidity (diabetes, dialysis-dependent chronic kidney disease, HIV infection, primary immunodeficiency, or 
Table 3. RA-related risk factors for tuberculosis among biological-naïve patients with RA.

\begin{tabular}{lccc}
\hline Exposure & TB Cases, $\mathrm{n}=31$ & Controls, $\mathrm{n}=122^{\text {aOR }(95 \% \mathrm{CI})^{*}}$ \\
\hline Treatment* & & & \\
$\quad$ Antimalarials & $6 / 30(20)^{\mathrm{a}}$ & $40 / 117(34)^{\mathrm{b}}$ & $0.5(0.2-1.4)$ \\
$\quad$ AZA & $5 / 30(17)^{\mathrm{a}}$ & $6 / 117(5)^{\mathrm{b}}$ & $3.8(1.1-13.8)$ \\
AZA the last year before TB & $4 / 30(13)^{\mathrm{a}}$ & $1 / 117(0.8)^{\mathrm{b}}$ & $17(1.8-161)$ \\
LEF & $5 / 30(17)^{\mathrm{a}}$ & $4 / 118(3)^{\mathrm{c}}$ & $6.0(1.5-24.7)$ \\
LEF the last year before TB & $4 / 30(13)^{\mathrm{a}}$ & $2 / 118(2)^{\mathrm{c}}$ & $8.6(1.5-50.5)$ \\
MTX & $21 / 31(68)$ & $88 / 121(74)^{\mathrm{a}}$ & $0.8(0.3-2.0)$ \\
PSL & $23 / 31(74)$ & $63 / 117(54)^{\mathrm{b}}$ & $2.5(1.0-6.0)^{* * *}$ \\
Sulfasalazine & $15 / 31(50)^{\mathrm{a}}$ & $50 / 116(43)^{\mathrm{d}}$ & $1.2(0.6-2.8)$ \\
No. of DMARDs, mean \pm SD (min-max) & $1.9 \pm 1.1(0-5)$ & $1.9 \pm 1.2(0-6)$ & $1.0(1.0-1.1)$ \\
COPD or asthma & $9 / 31(29)$ & $11 / 120(9)^{\mathrm{e}}$ & $3.9(1.5-10.7)$ \\
Smoking, n/N & $16 / 31^{\mathrm{d}}$ & $47 / 122^{\mathrm{f}}$ & $0.8(0.3-2.1)$ \\
\hline
\end{tabular}

Values are expressed as $\mathrm{n} / \mathrm{N}$ (\%) unless stated otherwise. ${ }^{\mathrm{a}}$ Missing data from 1 patient. ${ }^{\mathrm{b}}$ Missing data from 5 patients. ${ }^{\mathrm{c}}$ Missing data from 3 patients. ${ }^{\mathrm{d}}$ Missing data from 6 patients. ${ }^{\mathrm{e}}$ Missing data from 2 patients. ${ }^{\mathrm{f}}$ Missing data from 42 patients. ${ }^{*}$ Estimated from logistic regression models adjusted for sex and age. ${ }^{* *}$ Treatment for $\geq$ 4 consecutive weeks from RA diagnosis until TB diagnosis. ${ }^{* * *}$ Prednisolone additionally adjusted for concomitant DMARD use. aOR: adjusted OR; AZA: azathioprine; COPD: chronic obstructive pulmonary disease; DMARD: disease-modifying antirheumatic drug; LEF: leflunomide; MTX: methotrexate; PSL: prednisolone; TB: tuberculosis.

Table 4. Relative risk for tuberculosis among MTX-treated biologic-naïve patients with RA.

\begin{tabular}{|c|c|c|c|}
\hline Treatment With MTX* & TB Cases, $\mathrm{n}=21$ & Controls, $\mathrm{n}=88$ & $\mathrm{aOR}(95 \% \mathrm{CI})^{* *}$ \\
\hline Maximum dose, mg & $15.7 \pm 5.4(7.5-25)^{\mathrm{a}}$ & $14.1 \pm 4.9(7.5-25)^{b}$ & $0.9(0.8-1.0)$ \\
\hline Duration of treatment, weeks & $200 \pm 223(19-813)^{\mathrm{b}}$ & $241 \pm 1946(6-812)^{c}$ & $1.0(1.0-1.0)$ \\
\hline $\begin{array}{l}\text { Cumulative dose the last year } \\
\text { TB diagnosis, mg }\end{array}$ & $575 \pm 356(97.5-1040)^{d}$ & $622 \pm 283(120-1300)^{\mathrm{e}}$ & $1.0(1.0-1.0)$ \\
\hline $\begin{array}{c}\text { Weekly dose } 1 \text { year before TB } \\
\text { diagnosis, } \mathrm{mg} \text {, mean }[\mathrm{n}]\end{array}$ & $14.5[10]$ & $13.8[59]^{\mathrm{d}}$ & $0.97(0.83-1.12)$ \\
\hline
\end{tabular}

Values are expressed as mean \pm SD $(\min -\max )$ unless stated otherwise. ${ }^{a}$ Missing data from 4 patients. ${ }^{b}$ Missing data from 3 patients. ${ }^{\mathrm{c}}$ Missing data from 12 patients. ${ }^{\mathrm{d}}$ Missing data from 5 patients. ${ }^{\mathrm{e}}$ Missing data from 9 patients. * Treatment for $\geq 4$ consecutive weeks from RA diagnosis until TB diagnosis. ${ }^{* *}$ Estimated from logistic regression models adjusted for sex and age. aOR: adjusted OR; MTX: methotrexate; RA: rheumatoid arthritis; TB: tuberculosis.

Table 5. Relative risk for TB among PSL-treated biologic-naïve patients with RA.

\begin{tabular}{|c|c|c|c|}
\hline Treatment With PSL* & TB Cases, $\mathrm{n}=23$ & Controls, $n=63$ & $\mathrm{aOR}^{* *}(95 \% \mathrm{CI})$ \\
\hline Maximum dose, $\mathrm{mg}$ & $14.1 \pm 10.8(5-50)^{a}$ & $11 \pm 9.1(2.5-40)^{\mathrm{a}}$ & $1.04(0.98-1.10)$ \\
\hline Duration of treatment, weeks & $306 \pm 361(7-1426)^{a}$ & $297 \pm 324(4-1265)^{b}$ & $1.0(1.0-1.0)$ \\
\hline$\geq 15 \mathrm{mg} / \mathrm{d}$ PSL $\geq 1$ month ever before TB diagnosis, $\mathrm{n}(\%)$ & $3(10)$ & $7(5.7)$ & $0.9(0.1-8.3)$ \\
\hline $\begin{array}{l}\geq 15 \mathrm{mg} / \mathrm{d} \text { PSL } \geq 1 \text { month the last year before TB } \\
\quad \text { diagnosis, } \mathrm{n}(\%)^{\mathrm{a}}\end{array}$ & $2(6.5)$ & $1(0.8)$ & NA \\
\hline Mean daily dose 1 year before TB diagnosis, $\mathrm{mg}[\mathrm{n}]$ & $5.78[14]^{\mathrm{d}}$ & $5.66[40]^{\mathrm{d}}$ & $1.0(0.8-1.2)$ \\
\hline Mean daily dose at TB diagnosis, $\mathrm{mg}[\mathrm{n}]$ & $8.23[16]^{\mathrm{d}}$ & $5.12[41]$ & $1.2(1.05-1.5)$ \\
\hline
\end{tabular}

Values are expressed as mean \pm SD (min-max) unless stated otherwise. ${ }^{a}$ Missing data from 3 patients. ${ }^{b}$ Missing data from 9 patients. ${ }^{c}$ Missing data from 14 patients. ${ }^{\mathrm{d}}$ Missing data from 2 patients. ${ }^{*}$ Treatment for $\geq 4$ consecutive weeks from RA diagnosis until TB diagnosis. ${ }^{* *}$ Estimated from logistic regression models adjusted for sex, age, and DMARD medication. aOR: adjusted OR; DMARD: disease-modifying antirheumatic drug; RA: rheumatoid arthritis; PSL: prednisolone; TB: tuberculosis. 
malignancies) was associated with increased TB risk, and no patient suffered from a complicating comorbidity leading to discontinuation of RA treatment during the year before TB diagnosis.

There was no association between smoking and risk of $\mathrm{TB}$ (aOR $0.8,95 \%$ CI 0.3-2.1) but data were missing from 6 cases and 42 controls (Table 3).

Epidemiological risk factors for TB. Increased risk for TB was associated with a family history of TB (aOR 10.6, 95\% CI 2.9-39.3), a history of previous TB (aOR 9.2, 95\% CI 2.1-39.4), and being born outside Nordic countries (aOR 5.7, 95\% CI 1.2-27.1). Almost $90 \%$ of the cases (and the age-matched controls) were born before 1950, which is a known risk factor for TB in general in Sweden. Some epidemiological data were missing in the medical records, in particular for the controls (Table 6).

Taking both RA-associated (treatment with PSL and/or LEF) and epidemiological risk factors (born before 1950, former TB infection, known exposure of TB in the family, occupational history, and a history of living abroad in high-endemic areas) into account, the cases had at TB diagnosis a median of $3(1-6)$ noted risk factors for TB compared to a median of $2(0-4)$ in controls. The most common combination in cases was being born before 1950, having any suspected exposure of TB, and having been treated with oral corticosteroids for $\geq 4$ consecutive weeks during the course of RA.

\section{DISCUSSION}

In this population-based case-control study of $\mathrm{TB}$ occurring in biologic-naïve patients with RA, we found no association with commonly used csDMARDs and increased TB risk. Importantly, MTX, the most frequently used csDMARD in this study and in contemporary patients with RA in general, was not associated with an increased TB risk, regardless of duration of treatment and dose. Ever use of PSL was associated with a borderline increased TB risk, but we could not confirm previous findings of an association with the use of moderate-to-high doses of PSL $(\geq 15 \mathrm{mg})$.

Table 6. Epidemiological risk factors for TB in TB cases with biologic-naïve RA and matched RA controls.

\begin{tabular}{|c|c|c|c|}
\hline Risk Factor & $\begin{array}{l}\text { Cases, } \\
\mathrm{n}=31\end{array}$ & $\begin{array}{l}\text { Controls, } \\
\mathrm{n}=122\end{array}$ & $\begin{array}{c}\mathrm{aOR}^{*} \\
(95 \% \mathrm{CI})\end{array}$ \\
\hline Born before 1950 & $27(87.1)$ & $106(86.8)$ & - \\
\hline History of TB & $6(19.3)$ & $3(2.4)^{a}$ & $9.2(2.1-39.4)$ \\
\hline Family history of $\mathrm{TB}$ & $8(25.8)$ & $4(3.3)^{b}$ & $10.6(2.9-39.3)$ \\
\hline Work-related risk & $3(9.6)^{c}$ & $10(8.2)^{\mathrm{d}}$ & $0.9(0.2-3.8)$ \\
\hline Born in high-incidence country ${ }^{e}$ & $1(3.2)$ & $0(0)$ & NA \\
\hline Born outside Nordic countries ${ }^{f}$ & $4(12.9)$ & $3(2.4)$ & $5.7(1.2-27.1)$ \\
\hline $\begin{array}{l}\text { Lived/worked > } 3 \text { months } \\
\text { in high-incidence country }\end{array}$ & $1(3.2)$ & $0(0)$ & NA \\
\hline
\end{tabular}

${ }^{\mathrm{a}}$ Missing data from 119 patients. ${ }^{\mathrm{b}}$ Missing data from 118 patients. ${ }^{\mathrm{c}}$ Missing data from 9 patients. ${ }^{\mathrm{d}}$ Missing data from 29 patients. ${ }^{\mathrm{e}}>100$ cases/ 100,000.

${ }^{\mathrm{f}}$ Includes high-incidence countries. ${ }^{*}$ Estimated from logistic regression models adjusted for sex and age. Values are expressed as n (\%) unless stated otherwise. aOR: adjusted OR; NA: not applicable; RA: rheumatoid arthritis; TB: tuberculosis.
So far, results in studies on MTX and TB risk have been contradictory. Brode, et $a l^{11}$ reported no increased risk for TB, whereas Brassard, et al found an increased risk for TB (adjusted rate ratio 3.4, 95\% CI 1.8-6.4) linked to MTX treatment. Data on treatment duration and dose were, however, missing in both studies, and exposure was based on register information only. More recent studies focus mainly on MTX in combination with bDMARDs, which makes interpretation of the role of MTX difficult, although TB risk appears to be further increased when TNFi is used in combination with MTX or AZA as compared with $\mathrm{BDMARD}$ monotherapy regimens. ${ }^{24}$

Treatment with PSL during the course of RA was associated with a borderline increased risk of TB. We did not find any association between TB risk and maximum dose of PSL, the cumulative dose of PSL the year preceding TB diagnosis, nor with the use of PSL $\geq 15 \mathrm{mg} \geq 1 \mathrm{month}$, as indicated previously. ${ }^{25,26}$ Thus, our results suggest an increased risk of TB in corticosteroid-treated patients, but a clear cutoff between a safe and an unsafe dose could not be set. However, some other studies have reported a dose-dependent risk of TB with corticosteroids. In a study from Taiwan, the cumulative dose of corticosteroids was associated with an increased risk for TB. ${ }^{20}$ Similarly, an increased risk of TB associated with prescription of PSL, both for $\geq 7.5 \mathrm{mg} / \mathrm{d}$ vs $<7.5 \mathrm{mg}$, and $\geq 15 \mathrm{mg} / \mathrm{d}$ vs $<15 \mathrm{mg}$, was reported in a study from the UK. ${ }^{17}$

Based on few exposed cases, there were signals of increased TB risks in patients treated with LEF or AZA. Treatment with LEF has also been linked to an increased risk of TB in some previous studies, ${ }^{9,11,27,28}$ but the reason for this risk increase has not been specifically studied. One mode of action of LEF is an ability to suppress TNF- $\alpha,{ }^{29,30}$ and it could be speculated that this effect contributes to its association with $\mathrm{TB}$, in line with the well-described biological effect of TNF on maintenance of granulomas containing Mycobacterium tuberculosis in patients with LTBI. ${ }^{31,32}$

We have not identified other reports about TB risk in patients with RA treated with AZA, a sparsely used drug nowadays in the treatment of RA. In a study from 1975, AZA was reported to cause a depression of specific antimycobacterial resistance and also to affect in vitro cell-mediated immunological mechanisms of importance for infectious defense, ${ }^{33}$ but it is unclear if this could explain an association with TB and AZA in patients with RA. It should be noted that in our study (as in the previous studies), only a few of the patients had been exposed to LEF or AZA and that these TB cases constitute a small minority of all TB that occurs in biologic-naïve patients with RA. We also cannot exclude that patients with multicomorbidities were treated with these drugs instead of a bDMARD and that the increased risk of $\mathrm{TB}$ reflects other patient conditions rather than the exposure of drugs.

Obstructive pulmonary diseases, present in 9 (29\%) of the cases, was associated with a 3.9-fold increased risk of TB. An association between obstructive pulmonary disease and other pulmonary diseases (i.e., asthma, emphysema, and bronchitis) and TB has been described previously. ${ }^{17,34}$ In a Swedish population-based study from 2010, a diagnosis of chronic obstructive 
pulmonary disease (COPD) increased the risk for TB 3-fold compared to the general population. ${ }^{34}$ Several mechanisms could contribute to this association. Immunological mechanisms such as dysregulation of phagocytes ${ }^{35}$ together with inhaled corticosteroid treatment ${ }^{36}$ may increase the risk for mycobacterial infection. Malnutrition, a common problem in COPD, ${ }^{37}$ also increases the risk for $\mathrm{TB} .{ }^{38,39} \mathrm{COPD}$ and $\mathrm{TB}$ also share other risk factors such as tobacco smoking ${ }^{17,40}$ and diabetes..$^{35}$ Additionally, smoking (past or present) is common in patients with $\mathrm{RA},{ }^{41}$ and has been identified as an independent risk factor for TB in these patients. ${ }^{17}$ We did not find an increased TB risk associated with smoking, but due to missing data on smoking habits, especially among controls, this finding must be interpreted with caution.

We also noted an increase in RA activity before and during TB treatment among cases compared to controls. A higher RA disease activity during TB treatment may reflect a reduced antirheumatic treatment during this period, but an effect of the TB infection cannot be excluded, as the RA disease activity increased before TB diagnosis and initiation of TB treatment in many of the cases. Mycobacterial disease affects the immune system in different ways. The upregulation of toll-like receptors can lead to an increased production of proinflammatory cytokines such as TNF and interleukin 6 and $12,{ }^{42}$ which might induce more RA symptoms.

The majority of patients $(84 \%)$ presented with pulmonary $\mathrm{TB}$, and the main extrapulmonary site was lymph node. This is consistent with findings of TB in an elderly Swedish population and with 1 RA study from Japan, ${ }^{43}$ but is different from the increase in extrapulmonary TB reported after TNFi exposure. ${ }^{1}$ The $\mathrm{TB}$ prognosis was also comparable to $\mathrm{TB}$ prognosis in the general population, ${ }^{44}$ with $94 \%$ treatment success. Delay from symptom onset to TB diagnosis was close to 4 months. A long delay increases the risk of severe disease ${ }^{45}$ and greater transmission of infection. ${ }^{46}$

Epidemiological risk factors were reported in the medical records typically at TB diagnosis for the cases, but sparsely in the controls (without TB). These data are generally not asked for at the clinic until the patient is considered for bDMARD treatment or is diagnosed with TB. Apart from being born before 1950 , half of the cases had reported epidemiological risk factors suggestive of previous TB exposure. Only one of the cases had been screened for LTBI with a TST before onset of TB symptoms. To be born in Sweden before 1950 is a known general risk factor for $\mathrm{TB}$, reflecting the high prevalence of $\mathrm{TB}$ and a greater likelihood for TB exposure in Sweden during the first half of the last century. ${ }^{47}$

It is now generally accepted to screen for LTBI in rheumatic patients before the start of bDMARD or targeted synthetic DMARD treatment ${ }^{48}$ using a combination of data on epidemiological risk factors, chest radiograph, and immune-reactive tests. There is, however, growing support to also consider LTBI screening in biologic-naive patients. ${ }^{17}$ The effect of screening is also dependent on the incidence of TB. Although the overall incidence of TB is low in Sweden, the biologic-naïve patients with RA have a 4 -fold increased risk of TB compared to the general population, ${ }^{4}$ and represent a large number of the TB cases in the RA population. The majority of these TB cases are caused by reactivation of LTBI and thus potentially preventable by $\mathrm{TB}$ treatment if identified. It seems that several risk factors contribute to the increased $\mathrm{TB}$ risk in biologic-naive patients with RA and that prediction of TB based on RA-associated factors may be difficult in the individual patient. An important advantage of early screening for TB is to avoid influence of immune-modulating treatment on the test results. A number of studies have reported that immune-modulating drugs such as corticosteroids $s^{49,50}$ and $\mathrm{MTX}^{49}$ may cause both falsely negative results and indeterminate responses of TST and interferon- $\gamma$ release assays used to screen for LTBI.

Strengths of our study include the population-based setting using high-quality, nationwide registers for identification of cases and controls, combined with data from medical records for detailed clinical information. TB cases were identified from a large dataset, which enabled the study of this relatively rare outcome. Both RA and TB diagnoses were validated. Limitations include missing data in the medical records, which was more pronounced for controls than cases regarding epidemiological risk factors for TB. Data on socioeconomic risk factors were overall very limited and therefore not included in the analyses. The number of patients with some of the analyzed exposures such as treatment with LEF or AZA was low, making these results less robust and increasing the risk for bias and the chance to affect the results. Further, these patients might represent a cohort of RA patients with multicomorbidities with increased risk of TB. With few exposed individuals and risk of confounding factors, there is inherent uncertainty in the quantity and quality of these results and therefore should be cautiously interpreted.

In conclusion, several RA-associated risk factors such as treatment with LEF, AZA, or PSL, and concomitant obstructive lung disease may contribute to the increased TB risk in biologic-naïve patients with RA. We could not find any association with the use of moderate-to-high doses of PSL ( $\geq 15 \mathrm{mg}$ ) or treatment with MTX and an increased risk of TB. TB risk seems difficult to predict with precision in the individual biologic-naïve patient based on RA-associated risk factors. To further decrease the TB risk in patients with $\mathrm{RA}$, we therefore suggest considering $\mathrm{TB}$ screening in biologic-naïve patients.

\section{ONLINE SUPPLEMENT}

Supplementary material accompanies the online version of this article.

\section{REFERENCES}

1. Keane J, Gershon S, Wise RP, Mirabile-Levens E, Kasznica J, Schwieterman WD, et al. Tuberculosis associated with infliximab, a tumor necrosis factor alpha-neutralizing agent. N Engl J Med 2001;345:1098-104.

2. Bombardier C, Hazlewood GS, Akhavan P, Schieir O, Dooley A, Haraoui B, et al. Canadian Rheumatology Association recommendations for the pharmacological management of rheumatoid arthritis with traditional and biologic disease-modifying antirheumatic drugs: part II safety. J Rheumatol 2012;39:1583-602.

3. Singh JA, Saag KG, Bridges SL, Akl EA, Bannuru RR, Sullivan MC, et al. 2015 American College of Rheumatology guideline for the treatment of rheumatoid arthritis. Arthritis Rheumatol 2016; 68:1-26 
4. Arkema EV, Jonsson J, Baecklund E, Bruchfeld J, Feltelius N, Askling J; ARTIS Study Group. Are patients with rheumatoid arthritis still at an increased risk of tuberculosis and what is the role of biological treatments? Ann Rheum Dis 2015;74:1212-7.

5. Seong SS, Choi CB, Woo JH, Bae KW, Joung CL, Uhm WS, et al. Incidence of tuberculosis in Korean patients with rheumatoid arthritis (RA): effects of RA itself and of tumor necrosis factor blockers. J Rheumatol 2007;34:706-11.

6. Carmona L, Hernández-García C, Vadillo C, Pato E, Balsa A, González-Alvaro I, et al; EMECAR Study Group. Increased risk of tuberculosis in patients with rheumatoid arthritis. J Rheumatol 2003;30:1436-9.

7. Vadillo Font C, Hernández-García C, Pato E, Morado IC, Salido $\mathrm{M}$, Júdez $\mathrm{E}$, et al. [Incidence and characteristics of tuberculosis in patients with autoimmune rheumatic diseases]. [Article in Spanish] Rev Clin Esp 2003;203:178-82.

8. Breedveld FC, Weisman MH, Kavanaugh AF, Cohen SB, Pavelka $\mathrm{K}$, van Vollenhoven R, et al. The PREMIER study: a multicenter, randomized, double-blind clinical trial of combination therapy with adalimumab plus methotrexate versus methotrexate alone or adalimumab alone in patients with early, aggressive rheumatoid arthritis who had not had previous methotrexate treatment. Arthritis Rheum 2006;54:26-37.

9. Brassard P, Lowe AM, Bernatsky S, Kezouh A, Suissa S. Rheumatoid arthritis, its treatments, and the risk of tuberculosis in Quebec, Canada. Arthritis Rheum 2009;61:300-4.

10. Emery P, Breedveld F, van der Heijde D, Ferraccioli G, Dougados M, Robertson D, et al; Combination of Methotrexate and Etanercept in Early Rheumatoid Arthritis Trial Group. Two-year clinical and radiographic results with combination etanercept-methotrexate therapy versus monotherapy in early rheumatoid arthritis: a two-year, double-blind, randomized study. Arthritis Rheum 2010;62:674-82.

11. Brode SK, Jamieson FB, Ng R, Campitelli MA, Kwong JC, Paterson $\mathrm{JM}$, et al. Increased risk of mycobacterial infections associated with anti-rheumatic medications. Thorax 2015;70:677-82.

12. Matsuoka Y, Narukawa M. Comparison of serious adverse event profiles among antirheumatic agents using Japanese adverse drug event report database. Ther Innov Regul Sci 2018;52:339-47.

13. Brassard P, Kezouh A, Suissa S. Antirheumatic drugs and the risk of tuberculosis. Clin Infect Dis 2006;43:717-22.

14. Yoo HG, Yu HM, Jun JB, Jeon HS, Yoo WH. Risk factors of severe infections in patients with rheumatoid arthritis treated with leflunomide. Mod Rheumatol 2013;23:709-15.

15. Guirao-Arrabal E, Santos F, Redel-Montero J, Vaquero JM, Cantisán $S$, Vidal E, et al. Risk of tuberculosis after lung transplantation: the value of pretransplant chest computed tomography and the impact of mTOR inhibitors and azathioprine use. Transpl Infect Dis 2016;18:512-9.

16. Kim HA, Yoo CD, Baek HJ, Lee EB, Ahn C, Han JS, et al. Mycobacterium tuberculosis infection in a corticosteroid-treated rheumatic disease patient population. Clin Exp Rheumatol 1998;16:9-13.

17. Jick SS, Lieberman ES, Rahman MU, Choi HK. Glucocorticoid use, other associated factors, and the risk of tuberculosis. Arthritis Rheum 2006;55:19-26.

18. Agrawal PN, Gupta D, Aggarwal AN, Behera D. Incidence of tuberculosis among patients receiving treatment with oral corticosteroids. J Assoc Physicians India 2000;48:881-4.

19. Lai CC, Lee MT, Lee SH, Lee SH, Chang SS, Lee CC. Risk of incident active tuberculosis and use of corticosteroids. Int J Tuberc Lung Dis 2015;19:936-42.

20. Lai SW, Lin CL, Liao KF. Nation-based case-control study investigating the relationship between oral corticosteroids use and pulmonary tuberculosis. Eur J Intern Med 2017;43:53-7.
21. Ludvigsson JF, Andersson E, Ekbom A, Feychting M, Kim JL, Reuterwall C, et al. External review and validation of the Swedish national inpatient register. BMC Public Health 2011;11:450.

22. Forsberg L, Rydh H, Jacobsson A, Nyqvist K, Heurgren M. Kvalitet och innehåll i patientregistret. [Utskrivningar från slutenvården 1964-2007 och besök i specialiserad öppenvård (exklusive primärvårdsbesök) 1997-2007]. (Quality and content of the Patient Register)[Document in Swedish. Internet. Accessed May 20, 2021.] Available from www.socialstyrelsen.se/globalassets/ sharepoint-dokument/artikelkatalog/statistik/2009-125-15_ 200912515_rev2.pdf

23. Waldenlind K, Eriksson JK, Grewin B, Askling J. Validation of the rheumatoid arthritis diagnosis in the Swedish National Patient Register: a cohort study from Stockholm county. BMC Musculoskelet Disord 2014;15:432.

24. Lorenzetti R, Zullo A, Ridola L, Diamanti AP, Laganà B, Gatta $\mathrm{L}$, et al. Higher risk of tuberculosis reactivation when anti-TNF is combined with immunosuppressive agents: a systematic review of randomized controlled trials. Ann Med 2014;46:547-54.

25. Targeted tuberculin testing and treatment of latent tuberculosis infection. American Thoracic Society. MMWR Recomm Rep 2000;49:1-51.

26. Erkens CG, Kamphorst M, Abubakar I, Bothamley GH, Chemtob D, Haas W, et al. Tuberculosis contact investigation in low prevalence countries: a European consensus. Eur Respir J 2010;36:925-49.

27. Grover R, Dhir V, Aneja R, Arya V, Galle A, Marwaha V, et al. Severe infections following leflunomide therapy for rheumatoid arthritis. Rheumatology 2006;45:918-20.

28. Agrawal S, Sharma A. Dual mycobacterial infection in the setting of leflunomide treatment for rheumatoid arthritis. Ann Rheum Dis 2007;66:277.

29. Miceli-Richard C, Dougados M. Leflunomide for the treatment of rheumatoid arthritis. Expert Opin Pharmacother 2003;4:987-97.

30. Breedveld FC, Dayer JM. Leflunomide: mode of action in the treatment of rheumatoid arthritis. Ann Rheum Dis 2000;59:841-9.

31. Jacobs M, Samarina A, Grivennikov S, Botha T, Allie N, Fremond $\mathrm{C}$, et al. Reactivation of tuberculosis by tumor necrosis factor neutralization. Eur Cytokine Netw 2007;18:5-13.

32. Silva DAAD, Silva MVD, Barros CCO, Alexandre PBD, Timóteo RP, Catarino JS, et al. TNF-alpha blockade impairs in vitro tuberculous granuloma formation and down modulate Th1, Th17 and Treg cytokines. PLoS One 2018;13:e0194430.

33. Kvapilová M, Trnka L, Svejcar J, Pekárek J. Specific acquired resistance and activity of migration inhibition factor (MIF) in spleens of mice with chronic tuberculosis. Scand J Respir Dis 1975;56:305-11.

34. Inghammar M, Ekbom A, Engström G, Ljungberg B, Romanus V, Löfdahl CG, et al. COPD and the risk of tuberculosis-a population-based cohort study. PLoS One 2010;5:e10138.

35. O'Toole RF, Shukla SD, Walters EH. TB meets COPD: an emerging global co-morbidity in human lung disease. Tuberculosis 2015;95:659-63.

36. Brassard P, Suissa S, Kezouh A, Ernst P. Inhaled corticosteroids and risk of tuberculosis in patients with respiratory diseases. Am J Respir Crit Care Med 2011;183:675-8.

37. Mete B, Pehlivan E, Gülbaş G, Günen H. Prevalence of malnutrition in COPD and its relationship with the parameters related to disease severity. Int J Chron Obstruct Pulmon Dis 2018;13:3307-12.

38. Anuradha R, Munisankar S, Bhootra Y, Kumar NP, Dolla C, Babu S. Malnutrition is associated with diminished baseline and mycobacterial antigen - stimulated chemokine responses in latent tuberculosis infection. J Infect 2018;77:410-6. 
39. Hoyt KJ, Sarkar S, White L, Joseph NM, Salgame P, Lakshminarayanan $S$, et al. Effect of malnutrition on radiographic findings and mycobacterial burden in pulmonary tuberculosis. PLoS One 2019;14:e214011.

40. Chan ED, Kinney WH, Honda JR, Bishwakarma R, Gangavelli A, Mya J, et al. Tobacco exposure and susceptibility to tuberculosis: is there a smoking gun? Tuberculosis 2014;94:544-50.

41. Hafström I, Ajeganova S, Andersson ML, Bala SV, Bergman S, Bremander A, et al. A Swedish register-based, long-term inception cohort study of patients with rheumatoid arthritis - results of clinical relevance. Open Access Rheumatol 2019;11:207-17.

42. Hemmi H, Takeuchi O, Kawai T, Kaisho T, Sato S, Sanjo H, et al. A toll-like receptor recognizes bacterial DNA. Nature 2000; 408:740-5.

43. Ishiguro T, Takayanagi N, Kagiyama N, Yanagisawa T, Sugita Y. Characteristics of tuberculosis in patients with rheumatoid arthritis: a retrospective single-center study. Intern Med 2014;53:1291-8.

44. Holden IK, Lillebaek T, Seersholm N, Andersen PH, Wejse C, Johansen IS. Predictors for pulmonary tuberculosis treatment outcome in Denmark 2009-2014. Sci Rep 2019;9:12995.

45. Virenfeldt J, Rudolf F, Camara C, Furtado A, Gomes V, Aaby P, et al. Treatment delay affects clinical severity of tuberculosis: a longitudinal cohort study. BMJ Open 2014;4:e004818.
46. Golub JE, Bur S, Cronin WA, Gange S, Baruch N, Comstock GW, et al. Delayed tuberculosis diagnosis and tuberculosis transmission. Int J Tuberc Lung Dis 2006;10:24-30.

47. Winqvist N, Björk J, Miörner H, Björkman P. Long-term course of Mycobacterium tuberculosis infection in Swedish birth cohorts during the twentieth century. Int J Tuberc Lung Dis 2011; 15:736-40.

48. Hasan T, Au E, Chen S, Tong A, Wong G. Screening and prevention for latent tuberculosis in immunosuppressed patients at risk for tuberculosis: a systematic review of clinical practice guidelines. BMJ Open 2018;8:e022445.

49. Matulis G, Juni P, Villiger PM, Gadola SD. Detection of latent tuberculosis in immunosuppressed patients with autoimmune diseases: performance of a Mycobacterium tuberculosis antigen-specific interferon gamma assay. Ann Rheum Dis 2008;67:84-90.

50. Sargın G, Şentürk T, Ceylan E, Telli M, Çildağ S, Doğan H. TST, QuantiFERON-TB Gold test and T-SPOT.TB test for detecting latent tuberculosis infection in patients with rheumatic disease prior to anti-TNF therapy. Tuberk Toraks 2018;66:136-43. 